\title{
Scalability of Phase Change Materials in Nanostructure Template
}

\author{
Wei Zhang, ${ }^{1}$ Biyun L. Jackson, ${ }^{2}$ Ke Sun, ${ }^{3}$ Jae Young Lee, ${ }^{4}$ Shyh-Jer Huang, ${ }^{5}$ Hsin-Chieh Yu, \\ Sheng-Po Chang, ${ }^{5}$ Shoou-Jinn Chang, ${ }^{5}$ and Ya-Hong Xie ${ }^{1}$ \\ ${ }^{1}$ Department of Materials Science and Engineering, University of California, Los Angeles, Los Angeles, CA 90095, USA \\ ${ }^{2}$ Portland Technology Development, Intel Corporation, Hillsboro, OR 97124, USA \\ ${ }^{3}$ WaferTech LLC, Camas, WA 98607, USA \\ ${ }^{4}$ GLOBALFOUNDRIES, Inc., Milpitas, CA 95035, USA \\ ${ }^{5}$ Advanced Optoelectronic Technology Center, Institute of Microelectronics, Department of Electrical Engineering, \\ National Cheng Kung University, Tainan 701, Taiwan \\ Correspondence should be addressed to Ya-Hong Xie; yahong.xie@gmail.com
}

Received 23 September 2014; Accepted 11 December 2014

Academic Editor: Jimmy C. Yu

Copyright (C) 2015 Wei Zhang et al. This is an open access article distributed under the Creative Commons Attribution License, which permits unrestricted use, distribution, and reproduction in any medium, provided the original work is properly cited.

The scalability of $\mathrm{In}_{2} \mathrm{Se}_{3}$, one of the phase change materials, is investigated. By depositing the material onto a nanopatterned substrate, individual $\mathrm{In}_{2} \mathrm{Se}_{3}$ nanoclusters are confined in the nanosize pits with well-defined shape and dimension permitting the systematic study of the ultimate scaling limit of its use as a phase change memory element. $\mathrm{In}_{2} \mathrm{Se}_{3}$ of progressively smaller volume is heated inside a transmission electron microscope operating in diffraction mode. The volume at which the amorphous-crystalline transition can no longer be observed is taken as the ultimate scaling limit, which is approximately $5 \mathrm{~nm}^{3}$ for $\mathrm{In}_{2} \mathrm{Se}_{3}$. The physics for the existence of scaling limit is discussed. Using phase change memory elements in memory hierarchy is believed to reduce its energy consumption because they consume zero leakage power in memory cells. Therefore, the phase change memory applications are of great importance in terms of energy saving.

\section{Introduction}

Chalcogenide phase change materials (PCM) are a family of materials with prominent contrast in conductivity between amorphous state (highly resistive) and crystalline state (highly conductive) [1]. When used as nonvolatile memory elements, crystalline-to-amorphous phase transition is conventionally accomplished by Joule heating from an electrical current pulse, during which the material is heated to above melting temperature followed by rapid quenching leaving the material being frozen in the amorphous state. The opposite amorphous-to-crystalline transition is achieved by heating to below melting temperature followed by a gradual lowering of the temperature leading to the formation of crystalline state [2-4]. Using phase change memory elements as nonvolatile memory in memory hierarchy is believed to reduce its energy consumption because they consume zero leakage power in memory cells. This advantage makes phase change memory element a perfect candidate in the memory industry due to its excellent performance in energy consumption. Driven by Moore's law [5], the system functionality increases rapidly in the same size package with decreasing device dimensions. A question crucial to the technology development of phase change random access memories (PcRAM) is the ultimate scaling limit. Despite the fact that the phase transition of some PCM in bulk form is well studied by dedicated groups $[3,6-8]$ and the fact that ultimate limit of phase change in $\mathrm{Ge}_{2} \mathrm{Sb}_{2} \mathrm{Te}_{5}$ was reported [9], the size effects of $\mathrm{In}_{2} \mathrm{Se}_{3}$, another important candidate in the PCM family, have not been reported yet. Size effects start to play an important role as PCM devices scale further down to below $10 \mathrm{~nm}$. Under these nanometer scale dimensions-(a) neither the crystallization temperature nor the melting temperature is the same as those of bulk and (b) crystalline state needs to 


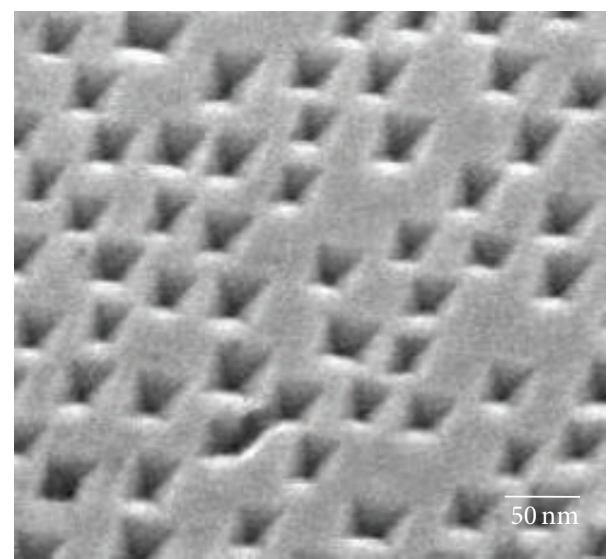

FIGURE 1: $35^{\circ}$ tilt SEM image of nanopit substrate after $\mathrm{KOH}$ etching.

have long range order-the amorphous and crystalline states may not be thermodynamically distinguishable any more. Therefore, a concern may be raised as to whether PCM has a size limit; that is, the crystalline state becomes unstable when the volume is reduced to a certain value. In this work, the scalability and ultimate size limit of $\mathrm{In}_{2} \mathrm{Se}_{3}$ are investigated. By depositing $\mathrm{In}_{2} \mathrm{Se}_{3}$ onto a unique nanopit substrate, size effects of the nano-volume $\mathrm{In}_{2} \mathrm{Se}_{3}$ confined in individual nanopits can be studied. The underlying physics of the diminishing of crystalline state is discussed.

\section{Experiment}

The fabrication of nanopit substrate is achieved by anisotropic $\mathrm{KOH}$ etching of Si. Figure 1 shows the SEM image of the nanopit substrate. Detailed process flow has been reported previously [10]. The tip of the nanopit is atomically sharp with the radius of curvature being less than $1 \mathrm{~nm}$ [11]. Amorphous $\mathrm{In}_{2} \mathrm{Se}_{3}$ with different nominal thickness is deposited onto the nanopit substrate by sputtering, followed by $\mathrm{SiO}_{2}$ capping layer to prevent the oxidation of $\mathrm{In}_{2} \mathrm{Se}_{3}$. Morphologies of the as-deposited films are examined by STEM. In order to determine the ultimate size limit, in situ heating TEM is used to observe the crystallization and melting behaviors of the nano-volume $\mathrm{In}_{2} \mathrm{Se}_{3}$.

\section{Results and Discussions}

3.1. Coverage of $\mathrm{In}_{2} \mathrm{Se}_{3}$ on Nanopit Substrate. Six different thicknesses $(2,3,5,8,23$, and $45 \mathrm{~nm})$ of $\mathrm{In}_{2} \mathrm{Se}_{3}$ were deposited on the nanopitted substrate. STEM studies revealed that two types of morphologies exist depending on the nominal film thickness. In Figure 2(a), bare Si nanopit substrate is shown. The contrast of the bare pit image is mainly from massthickness contrast since $\mathrm{Si}$ is the only element in the sample [12]. Similarly, the contrast of Figure 2(c), showing the $45 \mathrm{~nm}$ nominal film thickness of $\mathrm{In}_{2} \mathrm{Se}_{3}$ on nanopit substrate, is also contributed from mass-thickness contrast only since $\mathrm{In}_{2} \mathrm{Se}_{3}$ covers the whole sample surface. The difference between the morphologies of the two images is the size of the open area in the pit region. Smaller opening area for the $45 \mathrm{~nm}$ sample results from that the $\mathrm{In}_{2} \mathrm{Se}_{3}$ fills in the pits, coating the entire substrate uniformly and forming a conformal film. The $23 \mathrm{~nm}$ (not shown) sample is also conformably coated as well, with similar morphology as in Figure 2(c). On the other hand, samples of 2, 3, 5, and $8 \mathrm{~nm}$ nominal film thickness are distinct from the conformal case. In Figure 2(b), the film coverage of the $2 \mathrm{~nm}$ sample is shown (the morphologies of the 3,5, and $8 \mathrm{~nm}$ films are similar and are not shown here). We observed that there is lighter contrast from the pits region compared to the bare pit sample (Figure 2(a)). This indicates that the contrast is not only from mass-thickness contrast, but also from $Z$ (atomic number) contrast. The higher the $Z$ number of the material is, the more electrons get scattered at high angles into the High Angle Annular Dark Field (HAADF) detector [12]. Since $\mathrm{In}_{2} \mathrm{Se}_{3}$ is heavier than $\mathrm{Si}$, it should give a brighter contrast. In this sample, the pit region is relatively darker compared to the surface, which suggests that $\mathrm{In}_{2} \mathrm{Se}_{3}$ did not fill up the pits. For films with very thin nominal thickness, due to different interfacial energies between $\mathrm{In}_{2} \mathrm{Se}_{3} / \mathrm{Si}(100)$ and $\mathrm{In}_{2} \mathrm{Se}_{3} / \mathrm{Si}(111)$, materials deposited into the pits are very likely to be confined in there forming nanoclusters instead of conformal films. A schematic of film coverage on pits is also shown in Figure 2.

3.2. Phase Transition. The initial phase of the as-deposited $\mathrm{In}_{2} \mathrm{Se}_{3}$ is amorphous; thus, the diffraction pattern of the sample is a combination of halo amorphous rings from $\operatorname{In}_{2} \mathrm{Se}_{3}$ and diffraction spots from the Si substrate. Phase transition temperature is determined by the observation of single crystalline diffraction spots or polycrystalline diffraction rings appearing from crystalline $\mathrm{In}_{2} \mathrm{Se}_{3}$ as the sample is in situ heated. The sample temperature is first heated to around $90^{\circ} \mathrm{C}$, stabilized, and then annealed for 3 minutes. If no phase transition is observed in the time period, the sample temperature is then increased in $10^{\circ} \mathrm{C}$ increments. The process was repeated until phase transition is observed. Figure 3 shows the crystallization temperatures $\left(T_{x}\right)$ of the samples with different nominal film thickness. An increase in crystallization temperature is observed as the nominal film thickness decreases. Such crystallization behavior is also observed from the thin films $\mathrm{Ge}_{2} \mathrm{Sb}_{2} \mathrm{Te}_{5}, \mathrm{GeSb}$, and $\mathrm{Sb}_{2} \mathrm{Te}$ reported in another group's work [13]. For the sample with nominal thickness of $2 \mathrm{~nm}$ which translates to a volume of $5.3 \mathrm{~nm}^{3}$, no crystallization was observed. Therefore, $5.3 \mathrm{~nm}^{3}$ is believed to be the scaling limit of $\mathrm{In}_{2} \mathrm{Se}_{3}$. We speculate that the underlying physics of the scaling limit phenomenon is as follows. Thermodynamically, the driving force for crystallization is the reduction of the Gibbs free energy; that is, the amorphous phase is of higher energy which is at metastable state compared to the crystalline phase. However, for crystalline state $\operatorname{In}_{2} \mathrm{Se}_{3}$, the surface atoms are disordered or have higher energy than in the bulk due to the dangling bonds they have. This energy increase will be negligible for a bulk material with large volume. But with reducing volume or increasing surface to volume ratio, the higher energy of the surface atoms will become dominant in the total energy of the system. That means when the volume decreases to a certain value, which is the scaling limit, the disorderliness or the free energy of crystalline state is comparable to that of the amorphous state; 

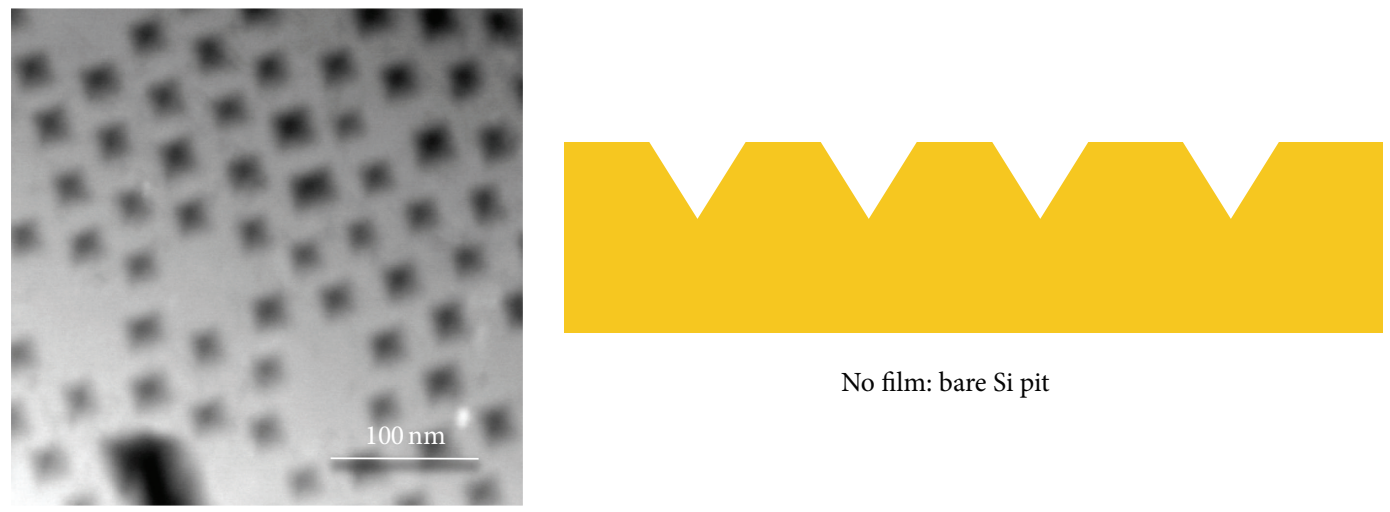

No film: bare Si pit

(a)
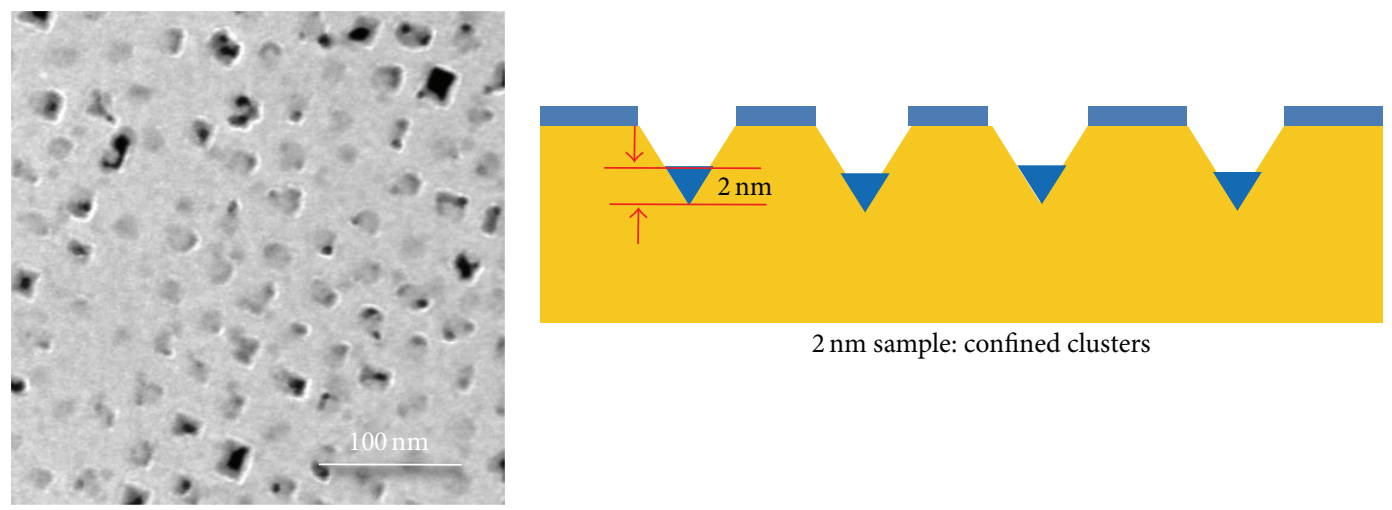

$2 \mathrm{~nm}$ sample: confined clusters

(b)
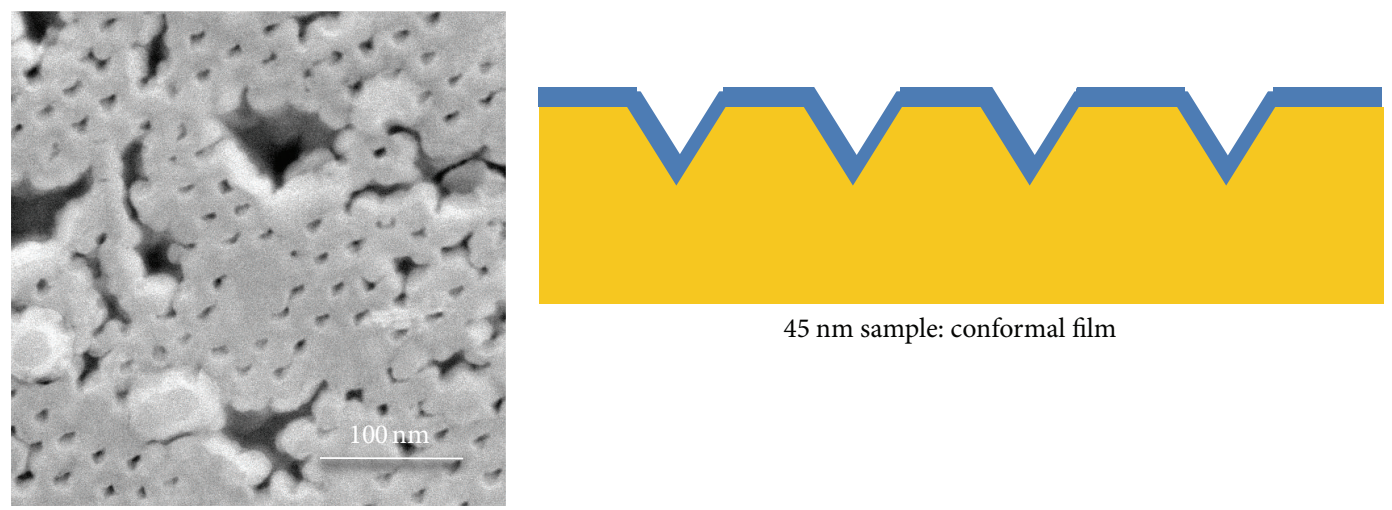

$45 \mathrm{~nm}$ sample: conformal film

(c)

FIGURE 2: STEM images of (a) bare nanopit Si substrate and (b) $2 \mathrm{~nm}$ and (c) $45 \mathrm{~nm} \mathrm{In}_{2} \mathrm{Se}_{3}$ deposited on Si nanopit substrate with cross section schematic of film coverage.

thus, the crystalline state diminishes. On the other hand, as we have already known from the working mechanism of $\mathrm{In}_{2} \mathrm{Se}_{3}$ phase transition, the crystallization temperature should be lower than the melting temperature. Therefore, the melting behavior of $\mathrm{In}_{2} \mathrm{Se}_{3}$ is also of interest as the crystallization temperature goes up with decreasing volume. The procedure for melting temperature measurement is similar to crystallization. The melting temperature is determined by the disappearance of polycrystalline diffraction rings. The sample is first heated to $280^{\circ} \mathrm{C}$ and then annealed for 3 minutes at the stabilized temperature. If no melting occurred, the sample temperature is increased by $10^{\circ} \mathrm{C}$. This process is repeated until melting is observed. As seen from Figure 4, the melting temperature $\left(T_{m}\right)$ decreases as the nominal film thickness decreases. As the volume of $\mathrm{In}_{2} \mathrm{Se}_{3}$ scales down, the melting temperature drops while the crystallization temperature rises. The $T_{m} / T_{x}$ ratio has dropped from 2.8 in bulk to 1.7 at $5 \mathrm{~nm}$ size. The melting temperature of the $2 \mathrm{~nm}$ nominal thickness sample cannot be measured because there were no polycrystalline diffraction rings observed throughout the entire heating process. This indicates that the decrease of melting temperature is also a potential cause for the scaling limit of $\mathrm{In}_{2} \mathrm{Se}_{3}$. 


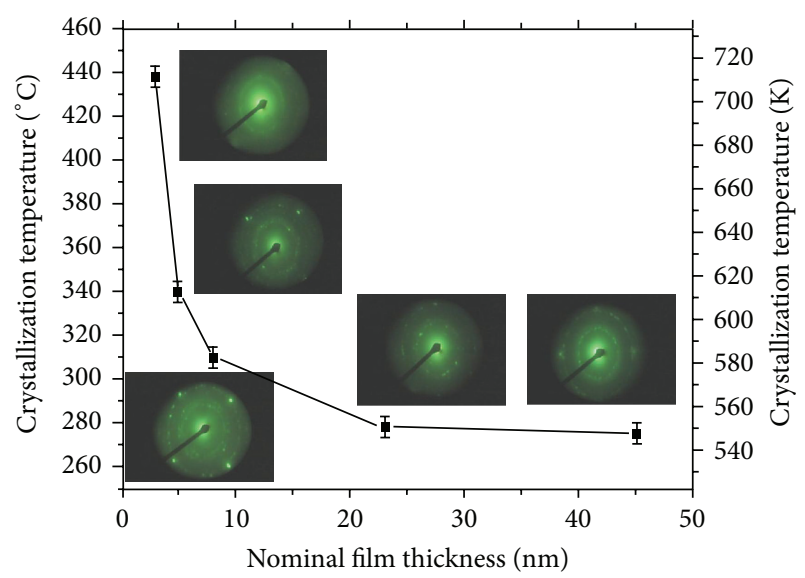

FIGURE 3: Crystallization temperature versus nominal film thickness of $\mathrm{In}_{2} \mathrm{Se}_{3}$ in nanopit substrate. Insets are diffraction pattern of the polycrystalline diffraction rings for each crystallization temperature.

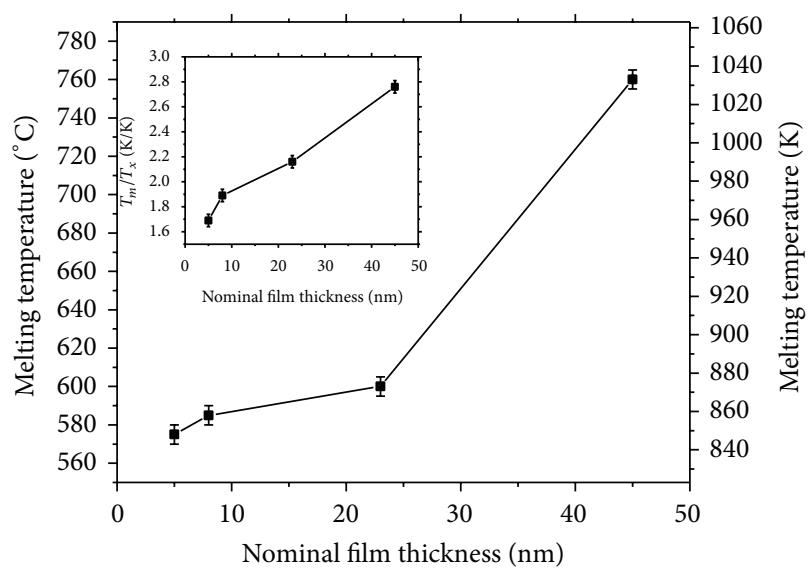

FIGURE 4: Melting temperature versus nominal film thickness of $\mathrm{In}_{2} \mathrm{Se}_{3}$ in nanopit substrate. Inset shows the ratio of $T_{m} / T_{x}$.

\section{Conclusion}

This study has shown that nanopit substrate is a unique pattern for nanosize PCM study. Film coverage on the nanopit substrate is not conformal when nominal film thickness of $\mathrm{In}_{2} \mathrm{Se}_{3}$ becomes very thin. $39 \%$ reduction of the ratio of $T_{m} / T_{x}$ is measured with reducing volume of $\mathrm{In}_{2} \mathrm{Se}_{3}$, and it is a possible cause for the elimination of phase transition. At $2 \mathrm{~nm}$ nominal film thickness, no crystallization is observed, which suggests that the corresponding volume of $5.3 \mathrm{~nm}^{3}$ is the scaling limit of $\mathrm{In}_{2} \mathrm{Se}_{3}$ concluded from this study. The diminishing of the crystalline state is believed to be due to the fact that the surface disorderliness dominates for small volume materials.

\section{Conflict of Interests}

The authors declare that there is no conflict of interests regarding the publication of this paper.

\section{Acknowledgments}

This work was supported in part by FAME, one of the six centers of STARnet, a Semiconductor Research Corporation program sponsored by MARCO and DARPA. Y. H. Xie acknowledges the support from Alexander von Humboldt Foundation Research Award that made this research possible.

\section{References}

[1] S. R. Ovshinsky, "Reversible electrical switching phenomena in disordered structures," Physical Review Letters, vol. 21, no. 20, pp. 1450-1453, 1968.

[2] H.-S. P. Wong, S. Raoux, S. Kim et al., "Phase change memory," Proceedings of the IEEE, vol. 98, no. 12, pp. 2201-2227, 2010.

[3] A. Redaelli, A. Pirovano, A. Benvenuti, and A. L. Lacaita, "Threshold switching and phase transition numerical models for phase change memory simulations," Journal of Applied Physics, vol. 103, no. 11, Article ID 111101, 2008.

[4] G. W. Burr, M. J. Breitwisch, M. Franceschini et al., "Phase change memory technology," Journal of Vacuum Science \& Technology B, vol. 28, no. 2, pp. 223-262, 2010.

[5] G. E. Moore, "Cramming more components onto integrated circuits," Electronics, vol. 38, pp. 114-117, 1965.

[6] M. Wuttig and N. Yamada, "Phase-change materials for rewriteable data storage," Nature Materials, vol. 6, no. 11, pp. 824-832, 2007.

[7] A. V. Kolobov, P. Fons, A. I. Frenkel, A. L. Ankudinov, J. Tominaga, and T. Uruga, "Understanding the phase-change mechanism of rewritable optical media," Nature Materials, vol. 3, no. 10, pp. 703-708, 2004.

[8] N. Yamada, E. Ohno, K. Nishiuchi, N. Akahira, and M. Takao, "Rapid-phase transitions of $\mathrm{GeTe}-\mathrm{Sb}_{2} \mathrm{Te}_{3}$ pseudobinary amorphous thin films for an optical disk memory," Journal of Applied Physics, vol. 69, no. 5, pp. 2849-2856, 1991.

[9] R. E. Simpson, M. Krbal, P. Fons et al., "Toward the ultimate limit of phase change in $\mathrm{Ge}_{2} \mathrm{Sb}_{2} \mathrm{Te}_{5}$," Nano Letters, vol. 10, no. 2, pp. 414-419, 2010.

[10] J. Y. Lee, K. Sun, B. Li, X. Wei, T. Russell, and Y.-H. Xie, "A method to fabricate a template with a long range ordered dense array of true nanometer scale pits," IEEE Transactions on Nanotechnology, vol. 10, no. 2, pp. 256-259, 2011.

[11] K. Sun, W. Zhang, B. Li et al., "Field emission tip array fabrication utilizing geometrical hindrance in the oxidation of Si," IEEE Transactions on Nanotechnology, vol. 11, no. 5, pp. 999-1003, 2012.

[12] D. B. Williams and C. B. Carter, Transmission Electron Microscopy: A Textbook for Materials Science, Springer, New York, NY, USA, 2009.

[13] S. Raoux, J. L. Jordan-Sweet, and A. J. Kellock, "Crystallization properties of ultrathin phase change films," Journal of Applied Physics, vol. 103, no. 11, Article ID 114310, pp. 114310-114316, 2008. 

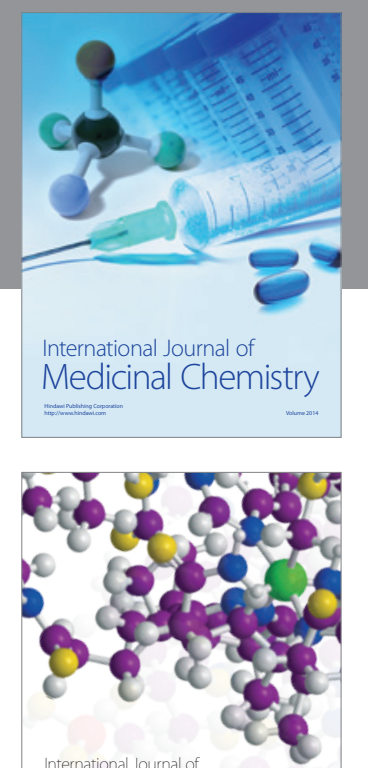

\section{Carbohydrate} Chemistry

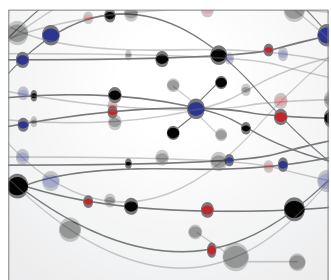

The Scientific World Journal
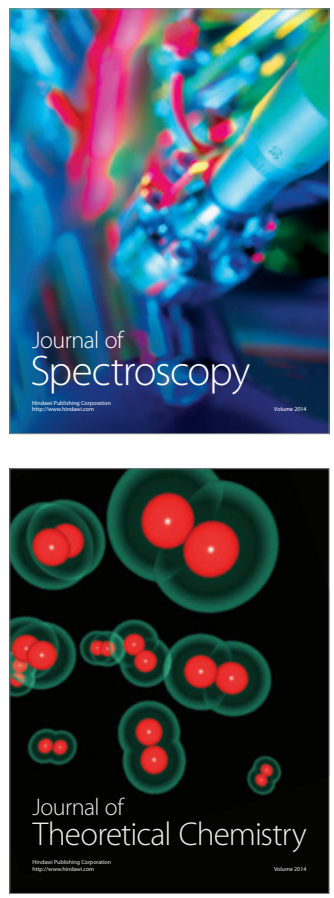
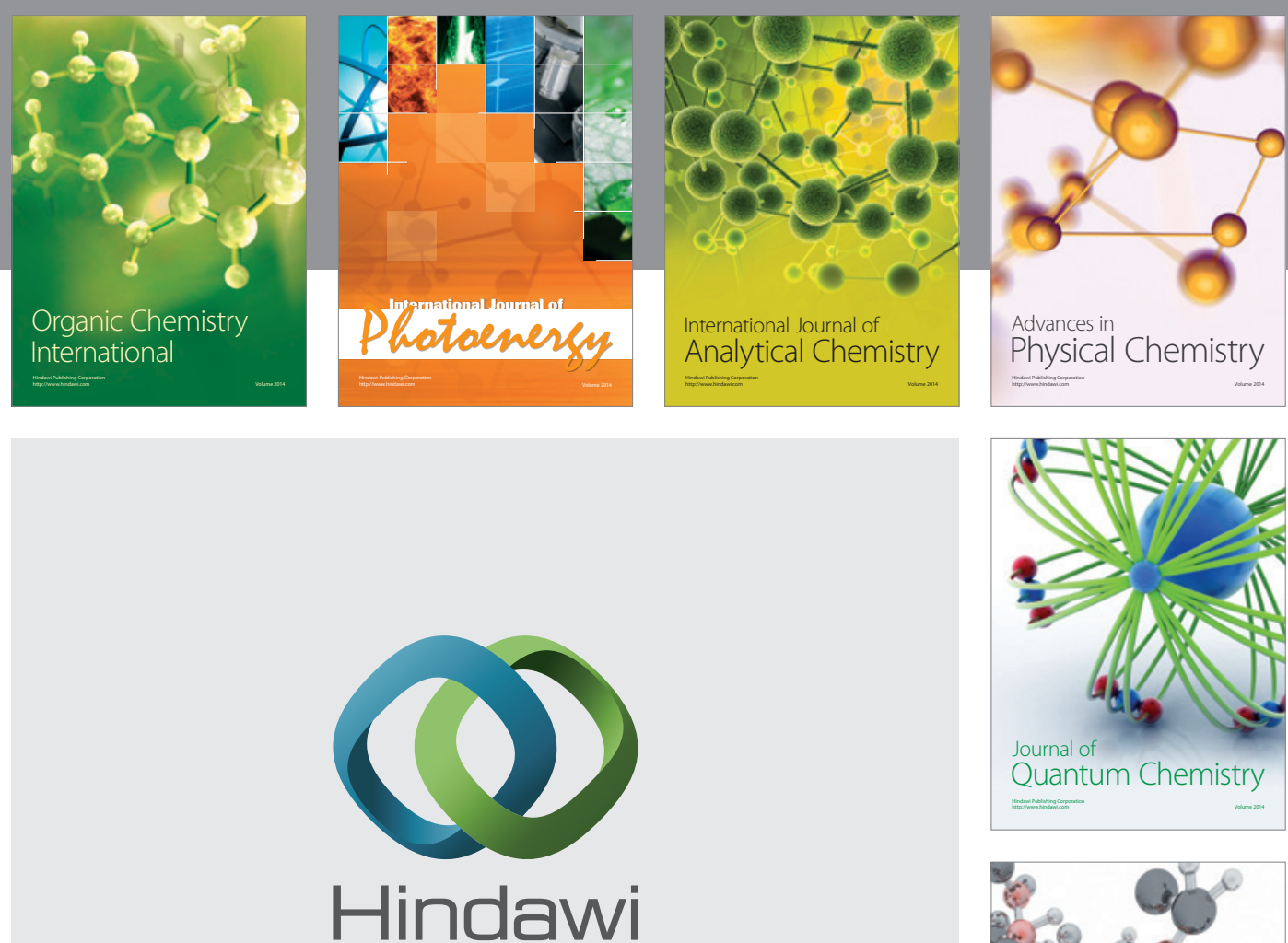

Submit your manuscripts at

http://www.hindawi.com

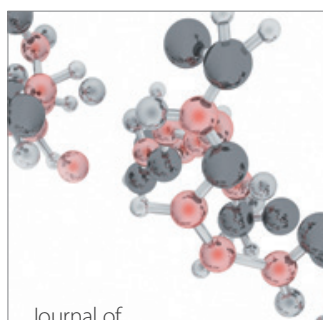

Analytical Methods

in Chemistry

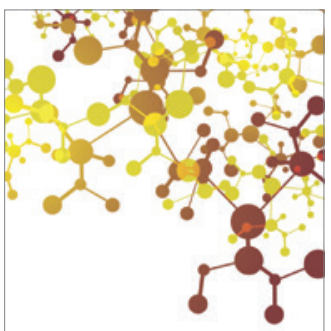

Journal of

Applied Chemistry

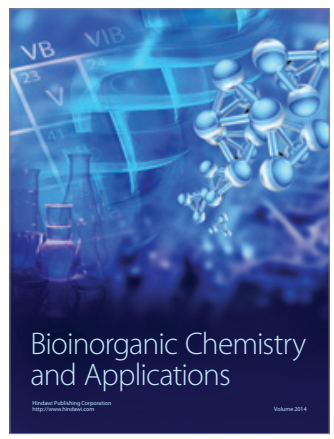

Inorganic Chemistry
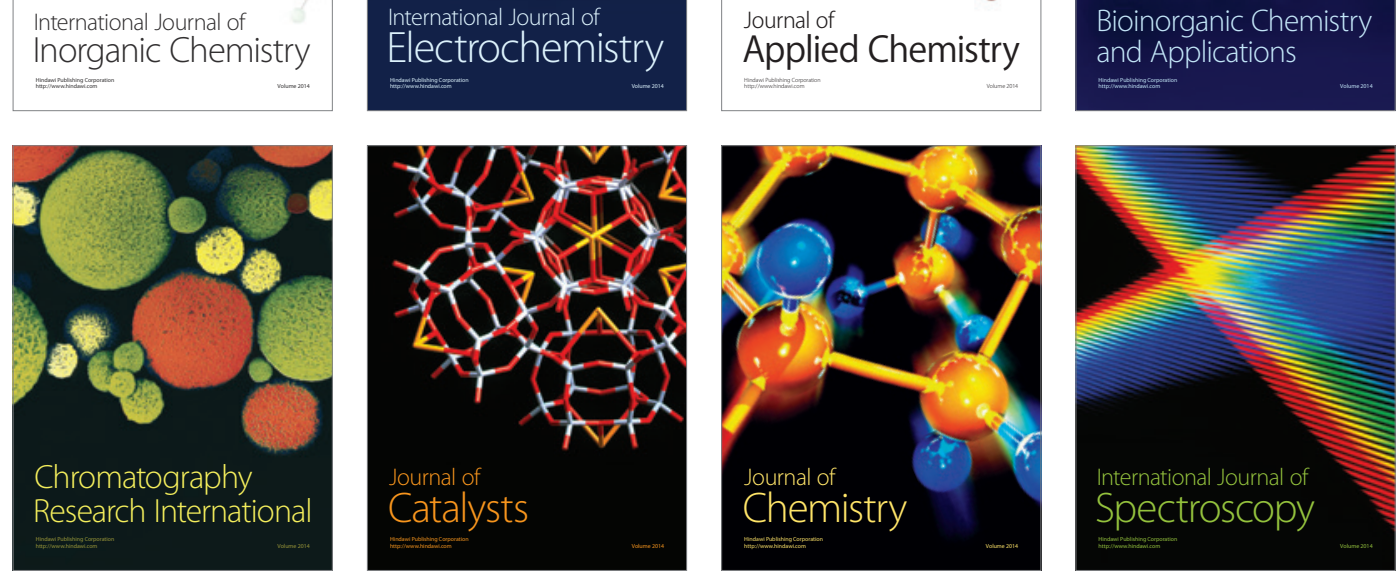